\title{
REVISIÓN
}

\section{Citocinas en la patogenia de la enfermedad celíaca}

\author{
Alberto J. Leóna, José A. Garrote ${ }^{a, b}$ y Eduardo Arranz \\ a Departamento de Pediatría e Inmunología, Instituto de Biología y Genética Molecular (IBGM). \\ Universidad de Valladolid. Valladolid. \\ b Unidad de Investigación. Hospital Clínico Universitario. Valladolid. España.
}

\begin{abstract}
La enfermedad celíaca se manifiesta por una enteropatía causada por la intolerancia al gluten, una familia de proteínas presente en el trigo y otros cereales. Tras la activación de los linfocitos $\mathrm{T}$ del intestino delgado en individuos predispuestos, se ponen en marcha mecanismos inflamatorios regulados por el balance entre citocinas inflamatorias de perfil Th1, como el interferón gamma (IFN $\gamma$ ), el factor de necrosis tumoral alfa (TNF $\alpha$ ), la interleucina (IL)-15 e IL-18, y otras reguladoras como el factor transformador del crecimiento beta (TGF $\beta$ ) e IL-10. Estas citocinas, además de incrementar el número de células del sistema inmunitario en la mucosa intestinal y el grado de activación, regulan la actividad de los factores de crecimiento epitelial y de las metaloproteinasas, moléculas encargadas de mantener y renovar la estructura de la mucosa, que en situaciones de inflamación provocan la lesión intestinal que conduce al síndrome de malabsorción.
\end{abstract}

Palabras clave: Enfermedad celíaca. Inflamación. Citocinas. Intestino.

\section{Cytokines in the pathogeny of celiac disease}

Celiac disease is manifested by an enteropathy caused by intolerance to gluten, a family of proteins found in wheat and other cereals. Following intestinal T-cell activation in predisposed individuals, different inflammatory mechanisms are triggered under the control of the cytokine balance including those with a pro-inflammatory Th1 pattern such as IFN $\gamma$, TNF $\alpha$, IL-15 and IL-18; and regulatory cytokines such as TGF $\beta$ and IL-10. These cytokines, besides increasing the intensity of the activation and the number of immune cells within the intestinal mucosa, regulate the activity of epithelial growth factors and metalloproteinases, a group of molecules involved in the maintenance and turnover of the intestinal mucosa structure; in inflammatory conditions, they also induce the intestinal lesion responsible for malabsorption syndrome.

Key words: Coeliac disease. Inflammation. Cytokines. Intestine.

La enfermedad celíaca (EC) o enteropatía por sensibilidad al gluten es la enfermedad inflamatoria intestinal más frecuente en nuestro entorno, con una prevalencia estimada de alrededor del 0,2-0,5\%, aunque a veces es asintomática o tiene una presentación atípica ${ }^{1,2}$. Cursa con malabsorción y diarrea secundaria a la disminución de la superficie de absorción de la mucosa del intestino delgado. La lesión es consecuencia de la activación de linfocitos T de la lámina propia mucosa en individuos genéticamente predispuestos como respuesta a la presencia de péptidos modificados de gluten ${ }^{3,4}$, componente proteico del trigo y otros cereales ${ }^{5}$. Los cambios en la arquitectura de la mucosa intestinal incluyen la infiltración celular, hiperplasia de criptas y pérdida de vellosidades, alteraciones que pueden tener distinta intensidad (grados 1 a 5 de Marsh), y que se recuperan hasta la normalidad histológica una vez eliminado el gluten de la dieta $^{6}$ (fig. 1).

Correspondencia: Dr. E. Arranz.

Departamento de Pediatría e Inmunología. Instituto de Biología y Genética Molecular (IBGM). Universidad de Valladolid.

Ramón y Cajal, 7. 47005 Valladolid. España.

Correo electrónico: earranz@med.uva.es

Recibido el 17-1-2005; aceptado para su publicación el 2-3-2005.

\section{Base inmunológica de la enfermedad celíaca}

El sistema inmunitario está directamente implicado en la patogenia de la EC, donde los linfocitos $T$ tienen un papel fundamental en la regulación de la inmunidad intestinal frente al gluten ${ }^{7}$. Las células presentadoras de antígeno interaccionan y procesan los antígenos que llegan al intestino, en un contexto que puede determinar que ciertas poblaciones de linfocitos T reconozcan a un antígeno como dañino para el organismo y deba actuarse contra él (inflamación), o inocuo, en cuyo caso debe mantenerse la situación de normalidad en la mucosa intestinal (tolerancia) ${ }^{8}$. El reconocimiento de
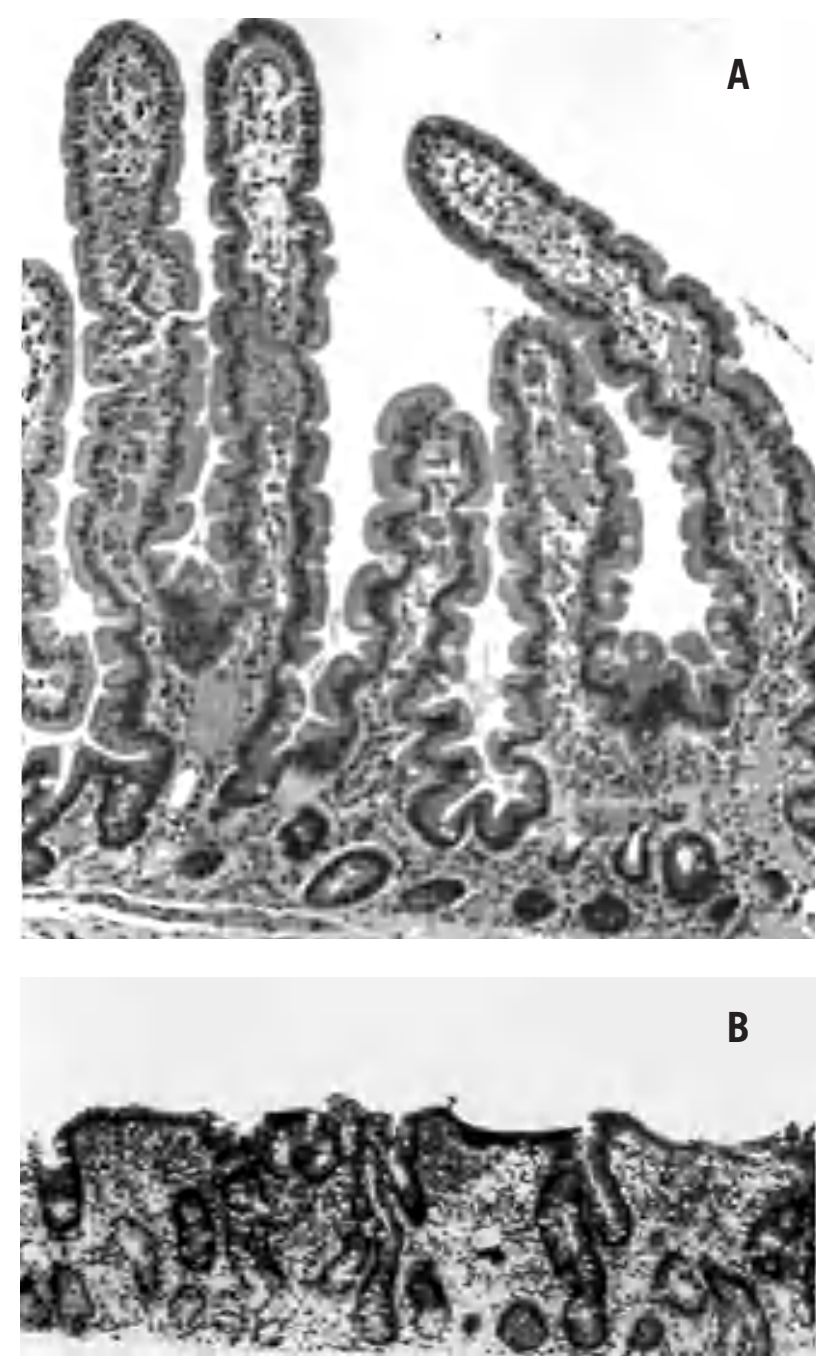

Fig. 1. Alteraciones en la arquitectura de la mucosa intestinal en la enfermedad celíaca. A: mucosa sana. B: atrofia vellositaria total (HE $\times 100)$. 
los péptidos de gluten por los linfocitos T reactivos de la lámina propia mucosa se lleva a cabo en el contexto de las moléculas del complejo mayor de histocompatibilidad, también conocido como HLA (anticuerpos leucocitarios humanos) de clase II, especialmente, HLA-DQ2 o DQ89,10. Para que se produzca este reconocimiento, los péptidos de gluten deben ser modificados antes por la enzima transglutaminasa tisular (TGt) mediante desaminación, que consiste en la sustitución de unos aminoácidos por otros con carga negativa ${ }^{11}$ (fig. 2). Esta enzima, además de tener una función esencial en el procesamiento del gluten en el intestino, es también el principal autoantígeno de los anticuerpos específicos para esta enfermedad ${ }^{12,13}$.

Mediante estudios de estimulación ex vivo con gluten a partir de biopsias obtenidas de pacientes celíacos en fase de remisión, se ha observado que las primeras señales de activación inmunitaria aparecen tan sólo 1 h después de haber entrado en contacto con el antígeno ${ }^{14}$. Ello sugiere la presencia de mecanismos tempranos de respuesta a señales de peligro, puestos en marcha por determinados péptidos sin capacidad de estimular a las células T reactivas al gluten, activándose posteriormente la respuesta celular específica mediada por estos linfocitos $T$ que reconocen péptidos de gluten en el contexto de las moléculas HLA-DQ2 o DQ8 ${ }^{15-17}$.

\section{Mecanismos de lesión}

Tras la activación de los linfocitos T específicos de gluten que asientan en la lámina propia de la mucosa intestinal de los pacientes celíacos, se liberan mediadores inflamatorios, como el interferón gamma (IFN $\gamma$ ), el factor de necrosis tumoral alfa (tumor necrosis factor alpha, TNF $\alpha$ ), la interleucina (IL) 15 , la IL-18 y el factor transformador del crecimiento beta (transforming growth factor beta, TGF $\beta$ ), entre otros $^{18,19}$. Estos mediadores causan diversos efectos sobre la mucosa, como la infiltración linfocitaria (tanto de la lámina propia como del epitelio), el aumento en la velocidad de degradación de la matriz extracelular ${ }^{20,21}$ y el aumento de los factores de crecimiento epitelial, como el factor transformador del crecimiento alfa (transforming growth factor alpha, $\mathrm{TGF} \alpha$ ) y factor de crecimiento de los queratinocitos (keratinocyte growth factor, KGF), que son causantes de la hiperplasia de las $\operatorname{criptas}^{22}$ (fig. 3).

En condiciones normales, las células de la estroma de la lámina propia mucosa secretan valores bajos de metaloproteinasas (matrix metalloproteinases, MMP), una familia de peptidasas con un átomo de cinc en su centro catalítico que degradan lentamente la matriz extracelular para su renovación ${ }^{23,24}$. También producen inhibidores de metaloproteinasas (tissue inhibitors of metalloproteinases, TIMP) que, junto a ciertos inhibidores, como la $\alpha 2$-macroglobulina, evitan el exceso de actividad enzimática. Las citocinas inflamatorias inducen rápidamente una elevación en los valores de MMP, pero sin alterar la producción de sus inhibidores $^{16,20,21}$.

Otra vía por la que las citocinas podrían verse indirectamente implicadas en los cambios observados en el intestino es a través de sus efectos en la producción de factores de crecimiento epitelial, como el $\mathrm{KGF}^{22}$, por las células de la estroma, que actúa específicamente sobre las células del epitelio intestinal ${ }^{25}$. Las células epiteliales producen sus propios mitógenos, como TGF $\alpha$ y factor de crecimiento epitelial (epithelial growth factor, EGF), y su liberación aumenta tras el daño tisular ${ }^{26}$. Es muy probable que la producción de esas moléculas y sus receptores pueda ser modulada por citocinas, aunque aún se necesitan más estudios para confirmar su importancia en el daño intestinal inducido por los linfocitos $\mathrm{T}^{27}$.

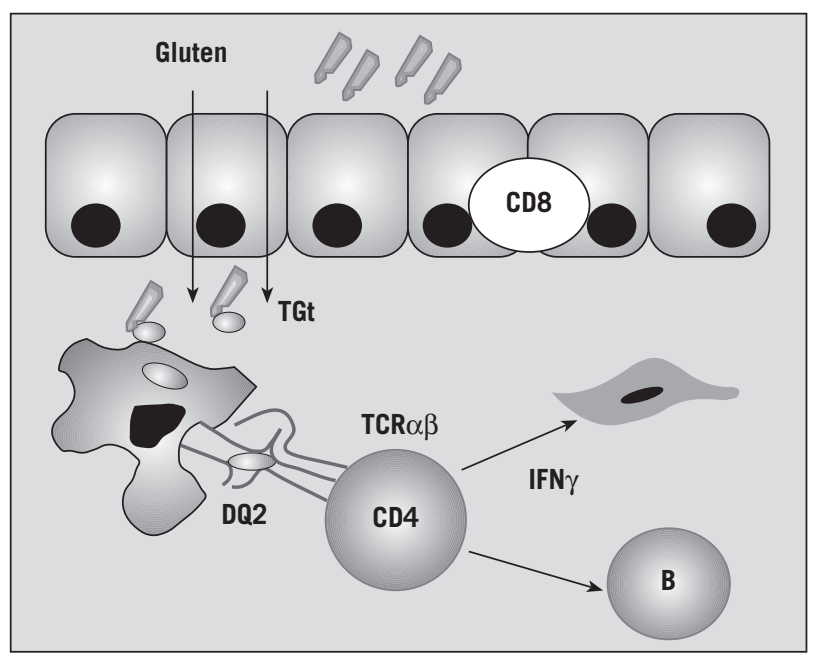

Fig. 2. Patogenia de la enfermedad celíaca.

Los linfocitos T CD4+ de la lámina propia mucosa tienen un papel central en la patogenia de la enfermedad celíaca, al controlar la respuesta inmunitaria local frente al gluten. Estos linfocitos T activados muestran un fenotipo Th1 con un patrón de producción de citocinas en el que predomina el IFN $\gamma$, capaz de actuar sobre otras células del estroma y proporcionar la ayuda necesaria a los linfocitos B para la síntesis de anticuerpos (antigliadina, antiendomisio y otros). TGt: transglutaminasa tisular; IFN : interferón gamma; DQ2: molécula codificada por los principales alelos de riesgo para la enfermedad celíaca; TCR $\alpha \beta$ : receptor de célula T de tipo $\alpha \beta$.

\section{Patogenia de la enfermedad celíaca}

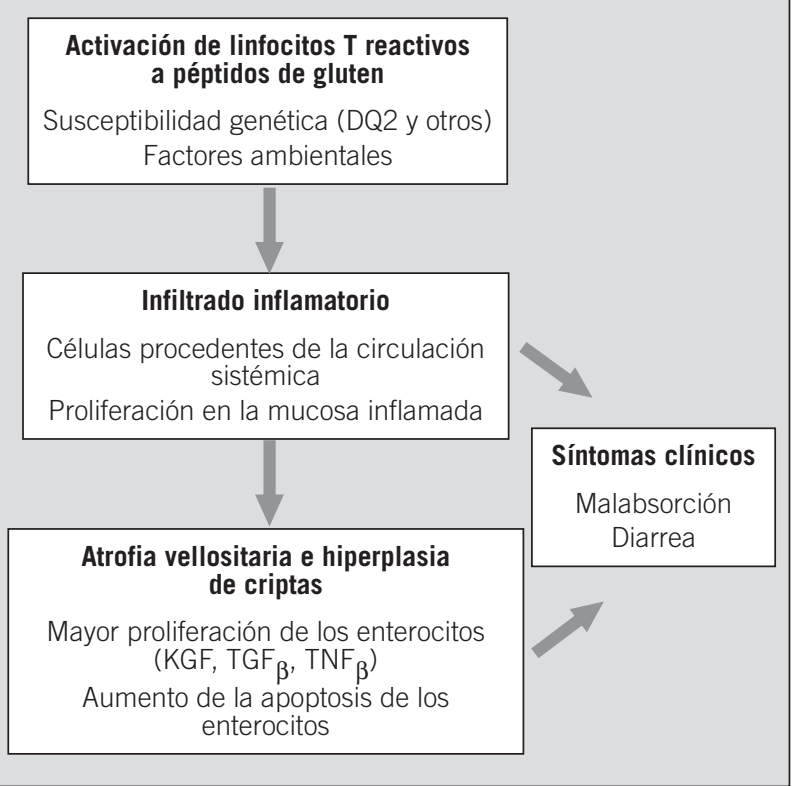

Fig. 3. Secuencia de acontecimientos que tienen lugar en la enfermedad celíaca. Los linfocitos T reactivos al gluten inician la respuesta inflamatoria que induce cambios en la arquitectura de la mucosa. Los síntomas clínicos se deben a cambios en la permeabilidad de la mucosa (producidos por el infiltrado inflamatorio), y a la disminución en la superficie de absorción (causados por la atrofia vellositaria). DQ2: principal alelo del HLA de clase I/ de riesgo en la enfermedad celíaca; KGF: factor de crecimiento de los queratinocitos; TGF $\beta$ : factor transformador del crecimiento beta; TNF : factor de necrosis tumoral beta.

\section{Relación de las citocinas con la lesión intestinal}

Los efectos conocidos de muchas citocinas explican gran parte de las alteraciones observadas en la EC, como el aumento en la proliferación de las criptas y la apoptosis de los enterocitos ${ }^{16}$, aunque ciertos aspectos todavía necesitan ser 


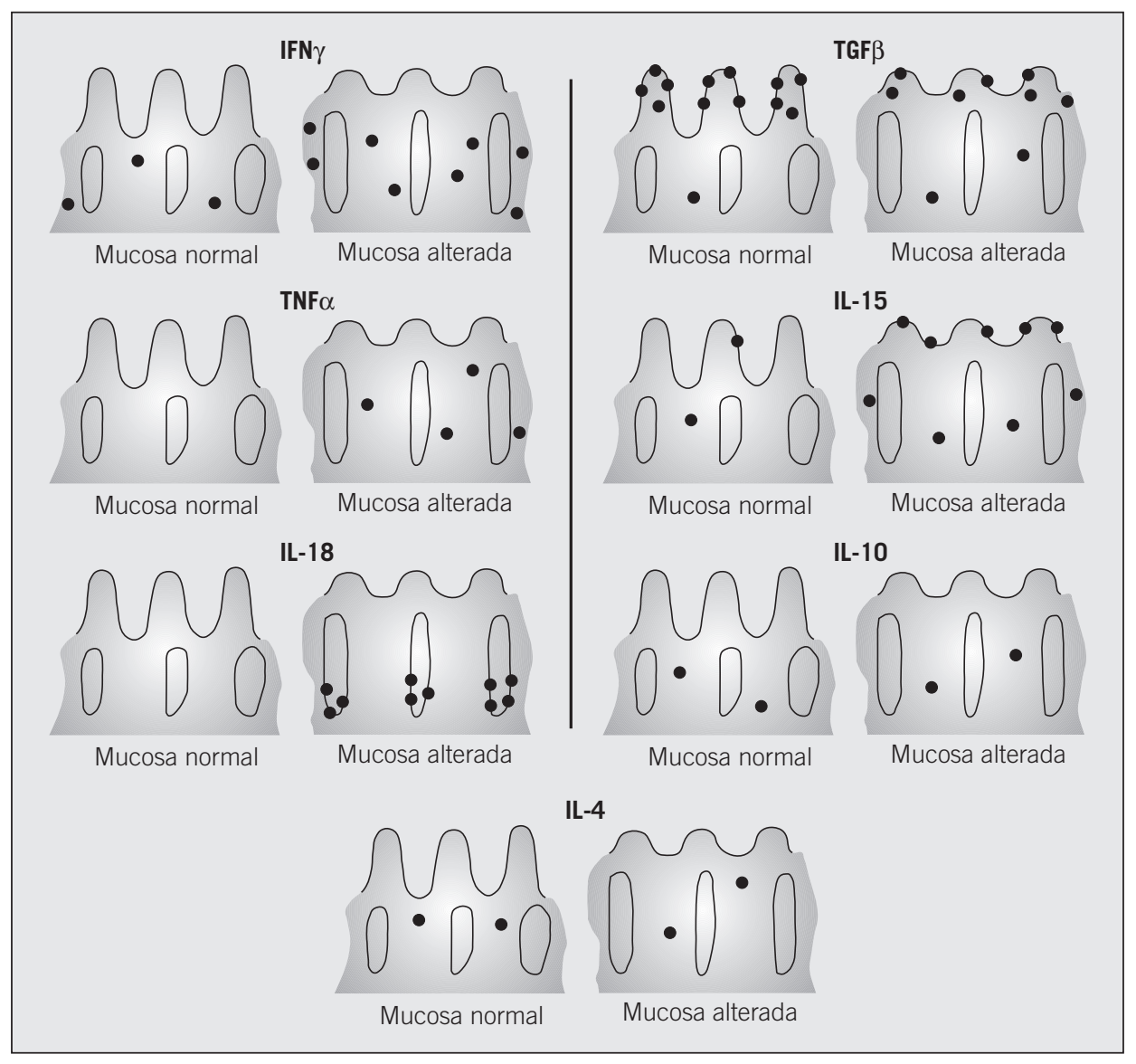

Fig. 4. Localización de las células productoras de citocinas en la mucosa intestinal, tanto en situación normal como en enfermedad celíaca en actividad.

IFN $\gamma$ : interferón gamma; TGFß: factor transformador del crecimiento beta; IL: interleucina; TNF $\alpha$ : factor de necrosis tumoral alfa. clarificados, como el aumento del número de linfocitos T intraepiteliales mucho antes de que se observen otras alteraciones en la mucosa del intestino, o su permanencia allí durante meses después de la retirada del gluten de la dieta y tras la recuperación de la normalidad histológica y funcional ${ }^{28}$

Los mecanismos de inflamación de la mucosa intestinal no son específicos de la EC, sino que también se ponen en marcha en otras situaciones. El grado y el tipo de alteración que sufre la mucosa dependen del patrón de citocinas y de su concentración local ${ }^{16}$. Por ejemplo, el IFN $\gamma$, además de estar aumentado en la EC ${ }^{19,28,29}$, también aparece en otras inflamaciones del intestino que presentan un grado de alteración mayor de la mucosa, como la enfermedad de Crohn y la colitis ulcerosa ${ }^{30}$, donde los valores de IFN $\gamma$ están mucho más elevados que en la EC (observación propia). Cuando se retira el gluten de la dieta en pacientes con EC se recupera la normalidad histológica y los valores de citocinas vuelven a la normalidad, y no hay diferencias entre los pacientes en remisión (con dieta sin gluten) y los controles sanos $^{19,29}$. Hasta el momento, en la EC en actividad no ha sido demostrada la posible correlación entre los valores locales de citocinas y el grado de atrofia vellositaria, o entre aquellos y los valores de anticuerpos antiendomisio ${ }^{29}$; por tanto, es necesario considerar otros factores además de las citocinas para explicar todos los acontecimientos que tienen lugar en la lesión de la EC.

La EC puede presentarse en dos momentos bien diferenciados: por un lado, en niños después de la introducción del gluten en la dieta, y por otro, en adultos que no habían manifestado previamente síntomas clínicos². Hasta el momen- to, ningún trabajo ha abordado específicamente este tipo de estudio, es decir, no se han determinado los valores de citocinas en ambos grupos y establecido las posibles diferencias, sino que cada estudio se centra en niños o adultos, o agrupa a todos los pacientes celíacos con independencia de la edad ${ }^{19}$. Sin embargo, el análisis de los distintos estudios publicados no permite establecer diferencias entre los valores de expresión de citocinas en biopsias de pacientes con EC, sean niños o adultos ${ }^{29}$.

\section{La enfermedad celíaca presenta un perfil de citocinas Th1}

Los estudios sobre los mediadores inflamatorios en la EC concluyen que hay un aumento en la estimulación en la mucosa intestinal de tipo Th1, mediado por IFN $\gamma$ y TNF $\alpha$, aunque coexisten con otros mediadores de perfil regulador, como el TGF $\beta^{13}$ (fig. 4). El compartimiento en el que se regula la actividad inflamatoria en la EC es la lámina propia mucosa, lugar en el que se encuentran los linfocitos T reactivos al gluten y donde se expresan las citocinas de perfil Th1 responsables del inicio o desarrollo de la inflamación $18,19,29$ (tabla 1 ).

Hay una gran variabilidad en la información disponible sobre las diferentes citocinas en la EC, y hay más datos acerca de las que se identificaron inicialmente, como IFN $\gamma$, TGF $\beta$ y TNF $\alpha^{19,29}$, mientras que otras de reciente descubrimiento, como IL-18 e IL-15, han sido objeto de estudio en un pequeño número de trabajos $28,37,38$. A la hora de comparar distintos estudios disponibles, otro punto a tener en cuenta es el que se refiere a los aspectos metodológicos, ya que los resultados obtenidos en algunas citocinas difieren 
TABLA 1

\section{Expresión de citocinas en la mucosa intestinal de pacientes con enfermedad celíaca (EC) en actividad y en los controles} sanos

\begin{tabular}{|c|c|c|}
\hline & Valores de ARNm (RT-PCR/hibridación in situ) & Localización en el tejido (inmunohistoquímica/hibridación in situ) \\
\hline \multirow[t]{2}{*}{$\mathrm{IFN} \gamma$} & EC actividad $>$ normal & Normal: expresión en la lámina propia, no hay presencia en el epitelio \\
\hline & $\begin{array}{l}\text { Valores en EC en remisión son los mismos } \\
\text { que en sanos } \\
\text { 19,22,28,29,31,32 }\end{array}$ & $\begin{array}{l}\text { EC actividad: mayores niveles en la lámina propia, y aparece en } \\
\text { el epitelio } 19,29,32,33\end{array}$ \\
\hline TGF $\beta$ & $\begin{array}{l}\text { ARNm: } E C \text { actividad }=\text { normal } \\
\text { Valores proteína: } E C \text { actividad }>\text { normal }{ }^{19,29,31,34}\end{array}$ & $\begin{array}{l}\text { Normal: expresión en el epitelio (sobretodo en la punta de las vellosidades } \\
\text { y poco en las criptas) y en la lámina propia } \\
\text { (especialmente en las zonas próximas al epitelio) } \\
\text { EC actividad: ligera disminución en el epitelio, aumento en lámina propia } \\
\text { (región subepitelial) }\end{array}$ \\
\hline TNF $\alpha$ & $\begin{array}{l}\text { EC actividad }=\text { normal } \\
\text { Valores proteína: } E \text { EC actividad }>\text { normal }{ }^{19,28,29,35} \\
\text { Aumento de ARNm tras estimulación in vitro }\end{array}$ & $\begin{array}{l}\text { Normal: expresión en la lámina propia, no en el epitelio } \\
\text { EC actividad: aumento en la lámina propia, no aparece en el epitelio }{ }^{32,33}\end{array}$ \\
\hline IL-12 & $\begin{array}{l}\text { EC actividad = normal } \\
\text { IL-12 (subunidad p40) ausente o valores muy bajos }{ }^{19,28,36}\end{array}$ & $\begin{array}{l}\text { Normal: no detectable } \\
\text { EC actividad: no detectable }{ }^{19,28,36}\end{array}$ \\
\hline IL-15 & $\begin{array}{l}\text { ARNm: EC actividad }=\text { normal } \\
\text { Valores proteína: } E C \text { actividad }>\text { normal } \\
\text { Hay aumento en los valores de proteína activa sin cambios } \\
\quad \text { en el ARNm debido a regulación postranscripcional28,37 }\end{array}$ & $\begin{array}{l}\text { Normal: no se detecta }{ }^{37} \text {, o expresión en células mononucleares de la lámina } \\
\text { propia y en enterocitos de vellosidades y } \text { criptas }^{28} \\
\text { EC actividad: expresión en pocas células mononucleares de la lámina propia }{ }^{37} \text {, } \\
\text { o aumento importante en la lámina propia y en los enterocitos }{ }^{28}\end{array}$ \\
\hline IL-18 & $\begin{array}{l}\text { ARNm: EC actividad }=\text { normal } \\
\text { Proteína activa: EC actividad > normal } \\
\text { IL-18 se produce de forma inactiva, su activación } \\
\text { por la caspasa-1 es independiente de } \\
\text { los valores de ARNm de IL-18 } 18^{38}\end{array}$ & $\begin{array}{l}\text { Normal: no detectable } \\
\text { EC actividad: expresión en las criptas, no detectable en la lámina propia } \\
\text { (observación propia, en preparación) }\end{array}$ \\
\hline IL-10 & $\begin{array}{l}\text { EC actividad }>\text { normal }^{29,39}, \text { aunque otros estudios } \\
\text { no observan diferencias entre ambos grupos }\end{array}$ & $\begin{array}{l}\text { Normal: expresión en la lámina propia, no detectable en epitelio } \\
\text { EC actividad: expresión en la lámina propia sin diferencias entre ambos } \\
\text { grupos }{ }^{40} \text {, o disminución en la lámina propia y expresión en el epitelio }{ }^{29}\end{array}$ \\
\hline IL-4 & $\begin{array}{l}\text { EC actividad }=\text { normal } \\
\text { Valores muy bajos en ambos grupos }{ }^{19,29,32}\end{array}$ & $\begin{array}{l}\text { Normal: presencia esporádica en la lámina propia, no en el epitelio } \\
\text { EC actividad: igual que normales }{ }^{19,32,33,40}\end{array}$ \\
\hline
\end{tabular}

En algunas citocinas que no presentan variaciones en sus valores de ARNm se han especificado los cambios en la cantidad de proteína activa, este último dato es el que tiene relevancia fisiológica. IL: interleucina, IFN $\gamma$ : interferón gamma; TGF $\beta$ : factor transformador del crecimiento beta; TNF $\alpha$ : factor de necrosis tumoral alfa.

según la técnica utilizada (ya sea estudio del ARN mensajero [ARNm] o de la proteína), y en los últimos años la sensibilidad y la precisión de las técnicas de estudio de ARNm han aumentado considerablemente.

\section{IFN $\gamma$ y TNF $\alpha$ están aumentados en la enfermedad celíaca activa}

El IFN $\gamma$ se definió inicialmente como un agente con actividad antiviral, aunque tiene otras funciones esenciales tanto en la inmunidad innata como en la inmunidad celular específica. En la inmunidad innata, su secreción depende de las células natural killer (NK) en respuesta al reconocimiento de ciertos componentes de microorganismos, así como en respuesta a la IL-12. En la inmunidad adaptativa, las células T producen IFN $\gamma$ en respuesta al reconocimiento del antígeno, fenómeno potenciado, a su vez, por la IL-12. Es la principal citocina que desencadena la actividad bactericida de los macrófagos, estimula la expresión de moléculas del complejo mayor de histocompatibilidad de clases I y II y de moléculas coestimuladoras en las células presentadoras de antígeno, como macrófagos o células dendríticas. Otro efecto del IFN $\gamma$ es inducir la diferenciación de las células T CD4+ vírgenes hacia el fenotipo Th1, inhibiendo por el contrario la proliferación de la subpoblación Th2 ${ }^{41,42}$. Se ha observado que el IFN $\gamma$ es uno de los mediadores más importantes en la inflamación en el intestino, tanto en la enfermedad en humanos como en los modelos experimentales en animales, y su expresión se ha relacionado directamente con el daño tisular en varios procesos inflamatorios del intestino ${ }^{43}$.

En la EC se ha observado que los linfocitos específicos de gluten, tanto los aislados de biopsias de pacientes como los clones celulares generados in vitro, producen un patrón de citocinas Th1 en el que el IFN $\gamma$ se encuentra siempre pre- sente $^{10,19}$. Se ha demostrado también que hay una relación entre la producción de citocinas por linfocitos $T$ reactivos al gluten y las alteraciones del epitelio intestinal. Esto se demostró al utilizar el sobrenadante de un cultivo de linfocitos T reactivos tras su estimulación con gluten para un segundo cultivo in vitro de biopsias intestinales obtenidas de individuos sanos, observándose que la altura de los enterocitos se reducía de una forma dependiente de la dosis de dicho sobrenadante, y este efecto podía ser bloqueado mediante anticuerpos neutralizantes anti-IFN $\gamma^{44}$.

En general, todos los estudios concluyen que el IFN $\gamma$ es detectable en condiciones normales y aumenta en la EC en fase de actividad. Los resultados obtenidos al estudiar la expresión de ARNm (por las técnicas de RT-PCR e hibridación in situ) coinciden con los de inmunohistoquímica en cortes de tejido, tanto en lo que se refiere al aumento de los valores totales de IFN $\gamma$ como en su localización tisular. Mientras que en el tejido sano el IFN $\gamma$ se expresa esporádicamente en células mononucleares de la lámina propia de muestras de tejido de pacientes con EC en actividad, la expresión de IFN $\gamma$ aumenta en la lámina propia, pero raramente en el epitelio. Las principales células productoras de IFN $\gamma$ son los linfocitos T y los macrófagos, aunque son estos últimos los que producen mayor cantidad en la EC ${ }^{29}$.

Los estudios de estimulación in vitro de biopsias de pacientes con EC en fase de remisión dan idea de los acontecimientos que tienen lugar en las primeras fases de la respuesta al gluten. Cuando se estimulan biopsias completas, se detecta un incremento de ARNm de IFN $\gamma$ a los $30 \mathrm{~min}, \mathrm{y}$ se alcanzan los valores máximos al cabo de 2-6 h, seguido de una reducción progresiva ${ }^{19}$.

El TNF $\alpha$, producido por macrófagos activados, interviene en múltiples actividades como la acumulación de neutrófilos, la formación de granulomas, la inducción de la expresión de 
LEÓN AJ, ET AL. CITOCINAS EN LA PATOGENIA DE LA ENFERMEDAD CELÍACA

moléculas de adhesión en el endotelio y otros muchos efectos ${ }^{42}$. Cuando el TNF $\alpha$ actúa sobre la mucosa intestinal incrementa de forma notable su permeabilidad ${ }^{45} \mathrm{y}$, al tener efectos citostáticos sobre las células epiteliales, puede inducir la apoptosis de los enterocitos cuando actúa sinérgicamente con el IFN $\gamma^{46,47}$. Tanto la expresión como la acción del TNF $\alpha$ está muy relacionada con el IFN $\gamma$, que actúan conjuntamente en muchos tipos de inflamación intestinal, como en el caso de la enfermedad de $\mathrm{Crohn}^{48}$ y la EC ${ }^{13}$.

No se ha conseguido demostrar un incremento en los valores de ARNm de TNF $\alpha$ cuando se estudia la biopsia completa de pacientes con EC en actividad ${ }^{19,28}$ o poblaciones de linfocitos $\mathrm{T}$ aislados de estas biopsias ${ }^{29}$. En algún caso, no se detecta el aumento de ARNm de TNF $\alpha$ en biopsias tras la instilación de péptidos de gluten directamente por vía rectal ${ }^{35}$. Sin embargo, en otro estudio sí se ha detectado un aumento de la expresión de ARNm de TNF $\alpha$ tras la estimulación in vitro de biopsias intestinales ${ }^{19}$.

La mayoría de los estudios realizados mediante inmunohistoquímica concluyen que el TNF $\alpha$ sólo se expresa en la lámina propia mucosa y no en el epitelio, y aumenta su expresión en la EC con respecto a los controles sanos $32,33,49$. Sin embargo, en otro estudio mediante citometría de flujo que utiliza células aisladas del intestino se ha observado una mayor expresión de TNF $\alpha$ en las biopsias de pacientes con EC en actividad en comparación con controles sanos, lo que se debía especialmente a la actividad de los linfocitos intraepiteliales, mientras que se mantenían los mismos valores en los linfocitos de la lámina propia ${ }^{50}$.

\section{Ausencia de IL-12 en la enfermedad celíaca en actividad}

La IL-12 es un mediador de la respuesta inmunitaria precoz frente a microorganismos intracelulares, así como un factor inductor fundamental de las respuestas de tipo Th1. La molécula de IL-12 activa es un heterodímero formado por las 2 subunidades (p35 y p40). Las principales fuentes de IL-12 son las células presentadoras de antígeno como macrófagos activados y células dendríticas. Hay muchas células que sintetizan la subunidad p35, pero sólo estas células presentadoras de antígeno profesionales sintetizan el componente p40 y, por tanto, la citocina biológicamente activa. La IL-12 proporciona una importante conexión entre la inmunidad innata y la inmunidad adaptativa, ya que es producida durante las reacciones de inmunidad innata y estimula respuestas inmunitarias adaptativas al estimular la función de presentación de antígeno ${ }^{51,52}$.

Debido a la importancia de esta citocina en la inducción de respuestas Th1, como es el caso de la enfermedad de Crohn, se pensó inicialmente que esta citocina también sería uno de los factores implicados en la EC. Se ha observado que la subunidad p40 no se expresa ni en el tejido sano ni en las muestras de pacientes con EC en actividad 28,36 , pero tampoco en las biopsias de pacientes con EC tras su estimulación in vitro con péptidos de gluten ${ }^{19}$. Sin embargo, no hay ningún trabajo que haya estudiado la presencia de p35 en el intestino de pacientes con EC. Puesto que se necesita la presencia simultánea de las 2 subunidades para la producción de IL-12 ${ }^{52}$, esta citocina no estaría presente durante las primeras fases tras la estimulación in vitro de biopsias de EC con péptidos de gluten ${ }^{19}$ ni en muestras de pacientes con EC en actividad ${ }^{36}$. Otro hecho que apoya la idea de que la IL-12 no se encuentra implicada en la patogenia de la EC es que los valores de activación de STAT-4, el principal factor de transcripción inducido por la IL-12, son similares tanto en los pacientes con EC como en los controles sanos ${ }^{53}$. La búsqueda de otros factores capaces de inducir la dife- renciación Th1 y la producción de IFN $\gamma$, especialmente en ausencia de IL-12, es una de las principales cuestiones que quedan por resolver en la inmunopatogenia de la EC ${ }^{11}$.

\section{Citocinas potenciadoras de respuesta Th1 en ausencia de IL-12}

La IL-15 es una citocina producida por las células mononucleares y, probablemente, por muchos otros tipos celulares como respuesta a infecciones virales, componentes bacterianos, como lipopolisacáridos (LPS), u otros factores que activan la inmunidad innata ${ }^{42}$. La función mejor documentada de la IL-15 es favorecer la proliferación y activación de las células $\mathrm{NK}^{54}$, y podría considerarse que los efectos de la IL-15 en la respuesta innata son equivalentes a los de la IL-2 en la respuesta inmunitaria adaptativa. La IL-15 también puede actuar como factor de crecimiento y supervivencia de las células T, especialmente para las células T CD8+ de memoria de vida larga ${ }^{55}$, por lo que podría estar relacionada con la supervivencia de los linfocitos específicos de memoria implicados en la inflamación intestinal. A diferencia de otras citocinas, la IL-15 está sujeta a una estricta regulación postranscripcional ${ }^{56}$. El ARNm de la IL-15 tiene 2 isoformas que presentan péptidos señal de distinta longitud, y mientras que una isoforma conduce a la vía secretora, la otra genera una proteína de localización intracelular, con distintas actividades biológicas ${ }^{57}$. Los múltiples efectos de la IL-15, así como los aspectos sobre su regulación han sido ampliamente recogidos en una revisión ${ }^{58}$.

La IL-15 podría ser uno de los mediadores responsables de que la activación inmunitaria en la lámina propia mucosa en la EC tenga también efectos sobre el epitelio, como lo demuestra el hecho de que los cambios epiteliales producidos en las biopsias de pacientes con EC estimuladas con péptidos de gliadina puedan ser bloqueados con anticuerpos neutralizantes de IL-15, y que la estimulación con IL-15 sólo tenga efecto sobre las biopsias de estos pacientes y no sobre el tejido normal ${ }^{37}$. Además, la IL-15 puede ser causante de la proliferación y la permanencia de los linfocitos intraepiteliales en esta localización ${ }^{28,59,60}$.

El aumento de IL-15 ha sido demostrado mediante inmunohistoquímica, a partir de biopsias de pacientes con EC en actividad en comparación con el tejido sano, aunque no se detecta un incremento del ARNm ${ }^{28,37}$. Esto se debe a que la IL-15 está sujeta a una fuerte regulación postranscripcional que impide su expresión en condiciones normales, y cuando se hace necesaria su producción se activan mecanismos postranscripcionales que generan suficiente proteína a partir de la misma cantidad de ARNm ${ }^{58}$.

Los resultados obtenidos por inmunohistoquímica en los dos únicos estudios publicados por el momento indican patrones de expresión de IL-15 diferentes. En uno de ellos ${ }^{37}$, en la EC en actividad se observa un pequeño número de células mononucleares positivas en la lámina propia mucosa, pero ninguna en el epitelio, mientras que en la EC en remisión y en el tejido sano no hay células positivas. Por el contrario, en el otro estudio $^{28}$ se observa la presencia esporádica de células positivas en la lámina propia y una expresión moderada en las vellosidades del tejido sano; mientras que en la EC en actividad hay una fuerte expresión en las células mononucleares de la lámina propia y en los enterocitos de las criptas. Además, en los pacientes con EC en remisión los valores de IL-15 en la lámina propia son menores que en los casos de enteropatía activa, pero superiores a los observados en el tejido sano.

La IL-18, inicialmente designada como factor inductor de IFN $\gamma$ (IFN $\gamma$ inducing factor) tiene una gran variedad de efectos biológicos, en especial la inducción de respuestas en 
células Th1. Estas funciones incluyen la proliferación de células T, el incremento de la actividad lítica de las células NK y el aumento de la síntesis de IFN $\gamma$. Por tanto, la IL-18 tiene funciones similares a las de la IL-12. Además, la IL-18 es capaz de promover la cascada inflamatoria al aumentar la liberación de TNF $\alpha$, IL-8 e IL-1 por células mononucleares de sangre periférica ${ }^{61}$. Al igual que la IL-1 $\beta$, la IL-18 se sintetiza como precursor que debe ser escindido por la enzima de conversión de la IL-1 $\beta$ (ICE) o caspasa-1 para generar la proteína biológicamente activa ${ }^{62,63}$.

Hasta el momento, se ha publicado un único estudio sobre la implicación de la IL-18 en la $\mathrm{EC}^{38}$, en el que se detecta la presencia de IL-18 inmadura (con un peso molecular de 24 $\mathrm{kDa}$ ) en muestras de pacientes con EC tanto en actividad como en remisión, y en tejido normal; mientras que la forma activa (18 kDa) aparece también en las muestras en fases de actividad y remisión, pero no en el tejido sano. En cuanto al principal activador de la IL-18, la enzima de conversión de la IL-1 $\beta$, presenta la misma actividad en todos los grupos estudiados, probablemente debido a la presencia de otros mecanismos de activación de la IL-18, además de la caspasa- $1^{64}$, que serían responsables de activar la IL-18 en la EC. La expresión de ARNm de IL-18 es constitutiva, y se han observado valores similares en los pacientes con EC en fase activa y en los controles ${ }^{38}$. Se ha confirmado que dicho ARNm se transcribe eficazmente, como demuestra el hecho de que la forma inmadura (24 kDa) esté presente en todas las muestras estudiadas. Se han descrito situaciones similares en otras enfermedades y en modelos experimentales en animales en los que la actividad de la IL-18 depende de la activación postranscripcional y no del aumento del ARNm ${ }^{65-67}$. Sin embargo, también hay situaciones en las que el aumento en la expresión de la forma madura de la IL-18 va acompañada de un incremento del ARNm, como en el caso de la enfermedad de Crohn ${ }^{68}$.

\section{TGF $\beta$ e IL-10 tienen función reguladora}

El TGF $\beta$ tiene una función inmunodepresora sistémica, al inhibir la proliferación de linfocitos B y T69, mientras que localmente actúa como agente proinflamatorio, promoviendo la acumulación de neutrófilos, macrófagos y linfocitos en el lugar de la inflamación ${ }^{70,71}$, e inhibiendo la producción de inmunoglobulinas junto a la inducción del cambio de isotipo hacia $\operatorname{lgA}^{72}$. En lo referente a la acción específica que ejerce el TGF $\beta$ sobre la mucosa intestinal, los estudios sobre tolerancia oral y la colitis inducidas experimentalmente en modelos animales sugieren que la inflamación gastrointestinal es el resultado de un balance entre las citocinas proinflamatorias, principalmente IFN $\gamma$, y otras de carácter opuesto, como TGF $\beta^{73}$. Asimismo, el TGF $\beta$ promueve la restitución del epitelio intestinal tras producirse un daño en la mucosa, estimulando el crecimiento y la diferenciación de células del mesénquima, como los fibroblastos ${ }^{74}$. También tiene un importante papel en el recambio de la matriz extracelular al aumentar la síntesis de elastina, fibronectina y de distintos tipos de colágeno, y reduce la actividad de las metaloproteinasas, al disminuir su expresión y aumentar la de sus inhibidores (TIMP) ${ }^{75}$. El TGF $\beta$ se produce en forma inactiva, uniéndose a proteínas de la matriz extracelular y liberándose únicamente tras la activación proteolítica ${ }^{76}$. Hay 3 isoformas $\left(\mathrm{TGF} \beta_{1}, \mathrm{TGF} \beta_{2}\right.$, y TGF $\beta_{3}$ ), con pocas diferencias funcionales entre ellas, de las cuales el $T G F \beta_{1}$ es la forma predominante en el ser humano ${ }^{34}$.

Se ha detectado la presencia de TGF $\beta$ tanto en biopsias intestinales de pacientes con EC en actividad como en controles sanos. Los datos de inmunohistoquímica muestran un claro aumento en la EC, aunque no se ha podido demostrar un incremento paralelo de los valores de ARNm, lo que coincide con estudios anteriores donde se ha observado que puede haber un aumento de la expresión de proteína sin alteraciones significativas en los valores de ARNm ${ }^{77}$. Varios trabajos muestran la presencia de ARNm de TGF $\beta$ tanto en tejido normal como en muestras de pacientes con EC en actividad, aunque sin aumentos estadísticamente significativos. Según Lionetti et $\mathrm{al}^{34}$, hay una tendencia al incremento no estadísticamente significativo, tal vez debido al pequeño número de muestras estudiado. Cuando se ha evaluado el ARNm en linfocitos T aislados tanto de la lámina propia mucosa como del epitelio intestinal, su detección ha sido positiva en tejido de pacientes con EC tanto en actividad como en remisión y en controles, sin hallarse diferencias entre los 3 grupos $^{29}$. Al estudiar las biopsias de pacientes con EC estimuladas in vitro con péptidos de gliadina no se han observado cambios en los valores de ARNm de TGF $\beta_{1}{ }^{19}$, y tampoco cuando se utilizan biopsias de colon de pacientes con EC tras la estimulación in vivo por vía rectal ${ }^{35}$. En un estudio mediante inmunohistoquímica se ha observado la presencia de TGF $\beta$ en el tejido sano (sin distinguir entre las distintas isoformas), abundante en la punta de las vellosidades y escasa en las criptas, aunque también en la lámina propia de la mucosa, principalmente de la zona subepitelial, donde se expresa por las células T y los macrófagos activados. En el intestino de pacientes con EC en fase activa, la expresión de TGF $\beta$ disminuye en el epitelio y aumenta en la lámina propia, principalmente en la región subepitelial ${ }^{34}$. Otros autores han examinado la presencia de $\mathrm{TGF} \beta_{1}$ y $\mathrm{TGF} \beta_{3}$, aunque sin determinar la importancia relativa de cada isoforma, observando un aumento de TGF $\beta_{1}$ en la lámina propia de muestras de pacientes con EC en actividad, mientras que se mantienen los valores bajos en el epitelio, y la expresión de TGF $\beta_{3}$ aumenta tanto en el epitelio como en la lámina propia mucosa ${ }^{33}$.

La IL-10 es una importante citocina inmunorreguladora que actúa sobre las células presentadoras de antígeno mediante la inhibición tanto de la síntesis de citocinas ${ }^{78}$ como de moléculas coestimuladoras ${ }^{79}$ y moléculas HLA de clase $\|^{80}$. Asimismo, la IL-10 actúa directamente sobre la proliferación y la diferenciación de las células T. La activación de células T $\mathrm{CD}^{+}$humanas con células presentadoras de antígeno alogénicas en presencia de IL-10 produce anergia de larga duración específica de antígeno en las células $T^{81}$. Además, la IL-10 induce la diferenciación de células $T$ reguladoras de tipo I (Tr1) que son capaces de suprimir la respuesta inmunitaria Th1 por medio de la secreción de IL-10 y TGF $\beta^{82}$. La alteración de células T reguladoras parece tener importancia en la patogenia de ciertas enfermedades autoinmunitaria en humanos ${ }^{83}$, y más concretamente en la EC, donde se ha observado que la IL-10 disminuye significativamente la formación de clones de células T específicas de gluten ${ }^{39}$. EI ARNm de IL-10 parece estar incrementado tanto en la EC en actividad como en las biopsias de pacientes en remisión estimuladas ex vivo con gluten ${ }^{29,39}$, aunque hay discrepancias con los resultados de otros trabajos ${ }^{19,40}$. El estudio más reciente ${ }^{39}$ muestra un incremento en los valores de ARNm en las biopsias de pacientes con EC en actividad y, además, sugiere que los daños producidos en la EC podrían explicarse por una alteración en el balance inhibición/estimulación de las citocinas de la mucosa intestinal expresado por la relación entre los valores de IL-10/IFN $\gamma$. Sin embargo, otro trabajo anterior no aprecia diferencias significativas en los valores de ARNm de IL-10 entre la EC en actividad y los controles sanos ${ }^{82}$, y tampoco se detecta su presencia en muestras de estos pacientes, ni después de la estimulación ex vivo con gluten ${ }^{19}$. 
LEÓN AJ, ET AL. CITOCINAS EN LA PATOGENIA DE LA ENFERMEDAD CELÍACA

En cuanto a la localización de la IL-10 en la mucosa, también hay discrepancias en los resultados entre los dos únicos trabajos que abordan esta cuestión. En un trabajo se observa la presencia de la IL-10 únicamente en la lámina propia (mediante inmunohistoquímica e hibridación in situ), pero no hay diferencias cuantitativas entre las muestras de pacientes con EC en actividad y de tejido sano ${ }^{40}$. En el otro se localiza la presencia de células positivas en la lámina propia de las muestras de tejido sano, mientras que en muestras de pacientes con EC en actividad la expresión de IL-10 aparece sólo en el epitelio ${ }^{29}$.

\section{Otras citocinas estudiadas en la enfermedad celíaca}

En condiciones normales, la mucosa intestinal puede expresar simultáneamente IFN $\gamma$ e IL-4, por lo que se ha estudiado la posibilidad de que una citocina Th2 esté implicada en la EC a pesar de ser un trastorno del perfil predominantemente Th1 ${ }^{18}$. Se ha observado la expresión de IL-4 en la mucosa intestinal normal en valores bajos, y la mayoría de los datos indican que en la EC en actividad o tras la estimulación de biopsias in vitro con péptidos de gluten, estos valores se mantienen bajos. De hecho, casi todos los estudios que cuantifican ARNm de IL-4 detectan valores bajos en algunas muestras ${ }^{19,29,31}$, excepto en un caso en el que se muestra un aumento de IL-4, mediante hibridación in situ, en el tejido de pacientes con EC en actividad comparado con el tejido sano ${ }^{32}$. En cuanto a la localización de la IL-4, casi todos los estudios concluyen que se expresa sólo en la lámina propia ${ }^{19,32,40}$, y sólo en un caso se observa la presencia tanto en el epitelio como en la lámina propia ${ }^{33}$. Los datos obtenidos por inmunohistoquímica indican que no hay aumento en la EC con respecto al tejido sano ${ }^{19,33,40}$, lo que coincide con los resultados de ARNm.

Además de la posible implicación de IL-15 e IL-18 53 , la búsqueda de otros mediadores capaces de inducir el aumento de IFN $\gamma$ en la EC en ausencia de IL-12 ha llevado a estudiar la implicación del IFN $\alpha$, que atrae actualmente la atención ${ }^{11}$ por ser una citocina implicada en la inmunomodulación frente a antígenos propios y externos ${ }^{84,85}$, capaz de inducir respuestas Th1 ${ }^{86,87}$. A partir de explantes de intestino fetal humano, se ha estudiado el efecto del IFN $\alpha$ sobre los valores de otras citocinas, y se ha observado que el IFN $\alpha$ por sí mismo no produce modificaciones, pero en combinación con anticuerpos anti-CD3 induce la hiperplasia de criptas de forma dependiente de la dosis y aumentan los valores de IFN $\gamma$, TNF $\alpha$ y KGF, este último probablemente implicado en la proliferación epitelial ${ }^{88}$. Además, se ha publicado el caso de un paciente que desarrolló EC tras recibir IFN $\alpha$ como tratamiento por presentar una leucemia mieloide crónica ${ }^{36}$. Esto sugiere un papel relevante de IFN $\alpha$ en la EC, aunque por el momento la única prueba experimental se reduce a la confirmación de la presencia de IFN $\alpha$ por la técnica de Western blot en una proporción de muestras de pacientes con EC en actividad, mayor que en el tejido sano ${ }^{36}$.

\section{Conclusiones}

El gluten produce la activación de una respuesta inmunitaria en la mucosa intestinal de los pacientes celíacos y, una vez que esta proteína se retira de la dieta, se recupera la normalidad histológica y funcional, aunque la hipersensibilidad a los péptidos tóxicos persiste de por vida. El modelo inmunopatogénico actual, según el cual la activación de linfocitos T específicos desencadena la expresión de la enfermedad, no consigue explicar todas las alteraciones encontradas y, ade- más, se sospecha la presencia de otros mecanismos de respuesta frente a ciertos péptidos de gluten no mediados por la inmunidad adaptativa.

Muchos de los mecanismos inflamatorios puestos en marcha en la EC son comunes a otros procesos intestinales, aunque en la EC el balance entre citocinas proinflamatorias y antiinflamatorias hace que las alteraciones sean moderadas y autolimitadas, por lo que este balance puede expresarse como la relación entre pares de citocinas, ya sea IFN $\gamma / T G F \beta$ o IFN $\gamma /$ IL-10. Resulta llamativa la ausencia de IL-12, por ser la principal responsable de la inducción y el mantenimiento de las respuestas Th1, aunque en el caso de la EC, otras citocinas podrían estar implicadas en este proceso. La IL-18, por ejemplo, tiene capacidad para inducir la producción de IFN $\gamma$ y presenta muchos efectos en común con la IL-12. Por otro lado, la IL-15, además de las funciones inicialmente asociadas con la inmunidad innata, está también implicada en la actividad inflamatoria de la lámina propia y tiene efectos directos sobre el epitelio de la mucosa intestinal.

A pesar de los avances producidos en los últimos años en el conocimiento de los mecanismos que regulan la inflamación en la mucosa intestinal en la EC, persisten multitud de incógnitas por resolver. Hasta el momento, no se ha hallado una relación directa entre los valores de citocinas y el grado de lesión de la mucosa, o la presencia de los autoanticuerpos característicos de la EC. Asimismo, quedan por esclarecer las vías de activación de la inflamación intestinal que, en ocasiones, producen una inflamación moderada y, en otras, conducen a alteraciones más profundas en la arquitectura de la mucosa, como en el caso de la enfermedad inflamatoria intestinal.

\section{Agradecimientos}

Agradecemos al Prof. Blanco-Quirós la revisión crítica del manuscrito y sus comentarios. El trabajo en nuestro laboratorio está financiado con ayudas del Instituto de Salud Carlos III, Ministerio de Sanidad (PI020895, 02/3068), Junta de Castilla y León (VA057/04), y Sweden Diagnostics afiliada a Pharmacia Diagnostics.

\section{REFERENCIAS BIBLIOGRÁFICAS}

1. Fasano A, Catassi C. Current approaches to diagnosis and treatment of celiac disease: an evolving spectrum. Gastroenterology. 2001;120:63651.

2. Farrell RJ, Kelly CP. Celiac sprue. N Engl J Med. 2002;346:180-8.

3. MacDonald TT, Spencer J. Evidence that activated mucosal T cells play a role in the pathogenesis of enteropathy in human small intestine. J Exp Med. 1988; 167:1341-9.

4. Sollid LM. Molecular basis of celiac disease. Annu Rev Immunol. 2000;18:53-81.

5. Van de Kamer JH, Weijers HA. Coeliac disease (V). Some experiments on the cause of the harmful effect of wheat gliadin. Acta Paediatr. 1955;44:465-9

6. Marsh MN. Gluten, major histocompatibility complex, and the small intestine. A molecular and immunobiologic approach to the spectrum of gluten sensitivity («celiac sprue»). Gastroenterology. 1992;102:330-54.

7. Maiuri L, Ciacci C, Ricciardelli I, Vacca L, Raia V, Auricchio S, et al. Association between innate response to gliadin and activation of pathogenic T cells in coeliac disease. Lancet. 2003;362:30-7.

8. Mowat AM. Anatomical basis of tolerance and immunity to intestinal antigens. Nat Rev Immunol. 2003;3:331-41.

9. Lundin KE, Scott H, Hansen T, Paulsen G, Halstensen TS, Fausa O, et al. Gliadin-specific, HLA-DQ(alpha $1^{*} 0501$, beta $1^{*} 0201$ ) restricted T cells isolated from the small intestinal mucosa of celiac disease patients. J Exp Med. 1993;178:187-96.

10. Nilsen EM, Lundin KE, Krajci P, Scott H, Sollid LM, Brandtzaeg P. Gluten specific, HLA-DQ restricted T cells from coeliac mucosa produce cytokines with Th1 or Th0 profile dominated by interferon gamma. Gut. 1995;37:766-76.

11. Sollid LM. Coeliac disease: dissecting a complex inflammatory disorder. Nat Rev Immunol. 2002;2:647-55. 
12. Dieterich W, Ehnis T, Bauer M, Donner P, Volta U, Riecken EO, et al. Identification of tissue transglutaminase as the autoantigen of celiac disease. Nat Med. 1997;3:797-801.

13. Schuppan D. Current concepts of celiac disease pathogenesis. Gastroenterology. 2000;119:234-42.

14. Maiuri L, Picarelli A, Boirivant M, Coletta S, Mazzilli MC, De Vincenzi M, et al. Definition of the initial immunologic modifications upon in vitro gliadin challenge in the small intestine of celiac patients. Gastroenterology. 1996;110:1368-78

15. Godkin A, Jewell D. The pathogenesis of celiac disease. Gastroenterology. 1998:115:206-10.

16. MacDonald TT, Bajaj-Elliott M, Pender SL. T cells orchestrate intestinal mucosal shape and integrity. Immunol Today. 1999;20:505-10.

17. Matzinger $P$. The danger model: a renewed sense of self. Science. 2002;296:301-5.

18. Troncone R, Gianfrani C, Mazzarella G, Greco L, Guardiola J, Auricchio $S$, et al. Majority of gliadin-specific T-cell clones from celiac small intestinal mucosa produce interferon-gamma and interleukin-4. Dig Dis Sci. 1998;43:156-61.

19. Nilsen EM, Jahnsen FL, Lundin KE, Johansen FE, Fausa O, Sollid LM, et al. Gluten induces an intestinal cytokine response strongly dominated by interferon gamma in patients with celiac disease. Gastroenterology. 1998;115:551-63.

20. Daum S, Bauer U, Foss HD, Schuppan D, Stein H, Riecken EO, et al. Increased expression of mRNA for matrix metalloproteinases- 1 and -3 and tissue inhibitor of metalloproteinases- 1 in intestinal biopsy specimens from patients with coeliac disease. Gut. 1999;44:17-25.

21. Pender SL, Tickle SP, Docherty AJ, Howie D, Wathen NC, MacDonald TT. A major role for matrix metalloproteinases in T cell injury in the gut. $J$ Immunol. 1997;158:1582-90.

22. Salvati VM, Bajaj-Elliott M, Poulsom R, Mazzarella G, Lundin KE, Nilsen EM, et al. Keratinocyte growth factor and coeliac disease. Gut. 2001;49:176-81.

23. Visse R, Nagase H. Matrix metalloproteinases and tissue inhibitors of metalloproteinases: structure, function, and biochemistry. Circ Res. 2003;92:827-39.

24. Pender SL, MacDonald TT. Matrix metalloproteinases and the gut - new roles for old enzymes. Curr Opin Pharmacol. 2004;4:546-50.

25. Finch PW, Rubin JS, Miki T, Ron D, Aaronson SA. Human KGF is FGFrelated with properties of a paracrine effector of epithelial cell growth. Science. 1989;245:752-5.

26. Murphy MS. Growth factors and the gastrointestinal tract. Nutrition. 1998;14:771-4.

27. Podolsky DK. Healing the epithelium: solving the problem from two sides. J Gastroenterol. 1997;32:122-6.

28. Mention JJ, Ben Ahmed M, Begue B, Barbe U, Verkarre V, Asnafi V, et al. Interleukin 15: a key to disrupted intraepithelial lymphocyte homeostasis and lymphomagenesis in celiac disease. Gastroenterology. 2003; $125: 730-45$

29. Forsberg G, Hernell O, Melgar S, Israelsson A, Hammarstrom S, Hammarstrom ML. Paradoxical coexpression of proinflammatory and downregulatory cytokines in intestinal T cells in childhood celiac disease. Gas troenterology. 2002;123:667-78.

30. Kmiec Z. Cytokines in inflammatory bowel disease. Arch Immunol Ther Exp (Warsz). 1998;46:143-55.

31. Lahat N, Shapiro S, Karban A, Gerstein R, Kinarty A, Lerner A. Cytokine profile in coeliac disease. Scand J Immunol. 1999;49:441-6.

32. Westerholm-Ormio M, Garioch J, Ketola I, Savilahti E. Inflammatory cytokines in small intestinal mucosa of patients with potential coeliac disease. Clin Exp Immunol. 2002;128:94-101.

33. Hansson T, Ulfgren AK, Lindroos E, DannAEus A, Dahlbom I, Klareskog L. Transforming growth factor-beta (TGF-beta) and tissue transglutaminase expression in the small intestine in children with coeliac disease. Scand J Immunol. 2002:56:530-7.

34. Lionetti P, Pazzaglia A, Moriondo M, Azzari C, Resti M, Amorosi A, et al. Differing patterns of transforming growth factor-beta expression in normal intestinal mucosa and in active celiac disease. J Pediatr Gastroenterol Nutr. 1999;29:308-13.

35. Chowers Y, Marsh MN, De Grandpre L, Nyberg A, Theofilopoulos AN, Kagnoff MF. Increased proinflammatory cytokine gene expression in the colonic mucosa of coeliac disease patients in the early period after gluten challenge. Clin Exp Immunol. 1997;107:141-7.

36. Monteleone G, Pender SL, Alstead E, Hauer AC, Lionetti P, McKenzie C, et al. Role of interferon alpha in promoting $T$ helper cell type 1 responses in the small intestine in coeliac disease. Gut. 2001;48:425-9.

37. Maiuri L, Ciacci C, Auricchio S, Brown V, Quaratino S, Londei M. Interleukin 15 mediates epithelial changes in celiac disease. Gastroenterology. 2000;119:996-1006.

38. Salvati VM, MacDonald TT, Bajaj-Elliott M, Borrelli M, Staiano A, Auricchio $S$, et al. Interleukin 18 and associated markers of $T$ helper cell type 1 activity in coeliac disease. Gut. 2002;50:186-90.

39. Salvati VM, Mazzarella G, Gianfrani C, Levings MK, Stefanile R, De Giulio B, et al. Recombinant human interleukin 10 suppresses gliadin dependent $T$ cel activation in ex vivo cultured coeliac intestinal mucosa. Gut. 2005;54:46-53.

40. Beckett CG, Dell'Olio D, Kontakou M, Przemioslo RT, Rosen-Bronson S, Ciclitira PJ. Analysis of interleukin-4 and interleukin-10 and their association with the lymphocytic infiltrate in the small intestine of patients with coeliac disease. Gut. 1996;39:818-23.
41. Boehm U, Klamp T, Groot M, Howard JC. Cellular responses to interferon-gamma. Annu Rev Immunol. 1997;15:749-95.

42. Abbas AK, Lichtman AH, Pober JS. Inmunología Celular y Molecular. 4. a ed. Madrid: Interamericana-McGraw-Hill; 2002.

43. MacDonald TT. Effector and regulatory lymphoid cells and cytokines in mucosal sites. Curr Top Microbiol Immunol. 1999;236:113-35.

44. Przemioslo RT, Lundin KE, Sollid LM, Nelufer J, Ciclitira PJ. Histological changes in small bowel mucosa induced by gliadin sensitive T lymphocytes can be blocked by anti-interferon gamma antibody. Gut. 1995;36:874-9

45. Gibson PR. Increased gut permeability in Crohn's disease: is TNF the link? Gut. 2004;53:1724-5.

46. Guy-Grand D, DiSanto JP, Henchoz P, Malassis-Seris M, Vassalli P. Small bowel enteropathy: role of intraepithelial lymphocytes and of cytokines (IL-12, IFN-gamma, TNF) in the induction of epithelial cell death and renewal. Eur J Immunol. 1998;28:730-44.

47. Kaiser GC, Polk DB. Tumor necrosis factor alpha regulates proliferation in a mouse intestinal cell line. Gastroenterology. 1997;112:1231-40.

48. Neurath MF. Mucosal immunity in Crohn's disease. Inflamm Bowel Dis. 2004;10 Suppl 1:S29-S31.

49. Przemioslo RT, Kontakou M, Nobili V Ciclitira PJ. Raised pro-inflammatory cytokines interleukin 6 and tumour necrosis factor alpha in coeliac disease mucosa detected by immunohistochemistry. Gut. 1994;35: $1398-403$.

50. O'Keeffe J, Lynch S, Whelan A, Jackson J, Kennedy NP, Weir DG, et al. Flow cytometric measurement of intracellular migration inhibition factor and tumour necrosis factor alpha in the mucosa of patients with coeliac disease. Clin Exp Immunol. 2001;125:376-82.

51. Watford WT, Moriguchi M, Morinobu A, O'Shea JJ. The biology of IL-12: coordinating innate and adaptive immune responses. Cytokine Growth Factor Rev. 2003;14:361-8

52. Trinchieri G. Interleukin-12 and the regulation of innate resistance and adaptive immunity. Nat Rev Immunol. 2003;3:133-46.

53. Monteleone I, Monteleone G, Del Vecchio BG, Vavassori P, Cucchiara S, MacDonald TT, et al. Regulation of the T helper cell type 1 transcription factor T-bet in coeliac disease mucosa. Gut. 2004;53:1090-5.

54. Mrozek E, Anderson P, Caligiuri MA. Role of interleukin-15 in the development of human CD56+ natural killer cells from CD34+ hematopoietic progenitor cells. Blood. 1996:87:2632-40.

55. Kanegane H, Tosato G. Activation of naive and memory T cells by interleukin-15. Blood. 1996;88:230-5.

56. Waldmann TA, Tagaya Y. The multifaceted regulation of interleukin-15 expression and the role of this cytokine in NK cell differentiation and host response to intracellular pathogens. Annu Rev Immunol. 1999;17: 19-49.

57. Tagaya Y, Kurys G, Thies TA, Losi JM, Azimi N, Hanover JA, et al. Generation of secretable and nonsecretable interleukin 15 isoforms through alternate usage of signal peptides. Proc Natl Acad Sci U S A. 1997; 94:14444-9.

58. Fehniger TA, Caligiuri MA. Interleukin 15: biology and relevance to human disease. Blood. 2001;97:14-32

59. Lai YG, Gelfanov V, Gelfanova V, Kulik L, Chu CL, Jeng SW, et al. IL-15 promotes survival but not effector function differentiation of CD8+ TCRalphabeta+ intestinal intraepithelial lymphocytes. J Immunol. 1999;163:5843-50.

60. Ebert EC. Interleukin 15 is a potent stimulant of intraepithelial lymphocytes. Gastroenterology. 1998;115:1439-45.

61. Dinarello CA. IL-18: A TH1-inducing, proinflammatory cytokine and new member of the IL-1 family. J Allergy Clin Immunol. 1999;103:11-24.

62. Ghayur T, Banerjee S, Hugunin M, Butler D, Herzog L, Carter A, et al. Caspase- 1 processes IFN-gamma-inducing factor and regulates LPS-induced IFN-gamma production. Nature. 1997;386:619-23.

63. Gu Y, Kuida K, Tsutsui H, Ku G, Hsiao K, Fleming MA, et al. Activation of interferon-gamma inducing factor mediated by interleukin-1beta converting enzyme. Science. 1997;275:206-9.

64. Tsutsui H, Kayagaki N, Kuida K, Nakano H, Hayashi N, Takeda K, et al. Caspase-1-independent, Fas/Fas ligand-mediated IL-18 secretion from macrophages causes acute liver injury in mice. Immunity. 1999;11:359-67.

65. Gracie JA, Forsey RJ, Chan WL, Gilmour A, Leung BP, Greer MR, et al. A proinflammatory role for IL-18 in rheumatoid arthritis. J Clin Invest. 1999;104:1393-401.

66. Lu H, Shen C, Brunham RC. Chlamydia trachomatis infection of epithelial cells induces the activation of caspase-1 and release of mature IL-18. J Immunol. 2000;165:1463-9.

67. Tomita T, Jackson AM, Hida N, Hayat M, Dixon MF, Shimoyama T, et al. Expression of Interleukin-18, a Th1 cytokine, in human gastric mucosa is increased in Helicobacter pylori infection. J Infect Dis. 2001;183:620-7.

68. Monteleone G, Trapasso F, Parrello T, Biancone L, Stella A, Iuliano R, et al. Bioactive IL-18 expression is up-regulated in Crohn's disease. J Immunol. 1999;163:143-7.

69. Lee G, Ellingsworth LR, Gillis S, Wall R, Kincade PW. Beta transforming growth factors are potential regulators of B lymphopoiesis. J Exp Med. 1987; 166:1290-9.

70. Reibman J, Meixler S, Lee TC, Gold LI, Cronstein BN, Haines KA, et al. Transforming growth factor beta 1 , a potent chemoattractant for human neutrophils, bypasses classic signal-transduction pathways. Proc Natl Acad Sci U S A. 1991;88:6805-9. 
LEÓN AJ, ET AL. CITOCINAS EN LA PATOGENIA DE LA ENFERMEDAD CELÍACA

71. McCartney-Francis NL, Wahl SM. Transforming growth factor beta: a matter of life and death. J Leukoc Biol. 1994;55:401-9.

72. Coffman RL, Lebman DA, Shrader B. Transforming growth factor beta specifically enhances IgA production by lipopolysaccharide-stimulated murine B lymphocytes. J Exp Med. 1989;170:1039-44.

73. Strober W, Kelsall B, Fuss I, Marth T, Ludviksson B, Ehrhardt R, et al. Reciprocal IFN-gamma and TGF-beta responses regulate the occurrence of mucosal inflammation. Immunol Today. 1997;18:61-4.

74. Pierce GF, Mustoe TA, Lingelbach J, Masakowski VR, Griffin GL, Senior $\mathrm{RM}$, et al. Platelet-derived growth factor and transforming growth factorbeta enhance tissue repair activities by unique mechanisms. J Cell Biol. 1989;109:429-40.

75. Barnard JA CRJ. Transforming growth factor [beta]. En: Walsh JH, Dockray GJ, editor. Gut peptides: Biochemistry and physiology. New York: Raven Press; 1993. p. 615-31

76. Nunes I, Gleizes PE, Metz CN, Rifkin DB. Latent transforming growth factor-beta binding protein domains involved in activation and transglutaminase-dependent cross-linking of latent transforming growth factorbeta. J Cell Biol. 1997;136:1151-63.

77. Letterio JJ, Roberts AB. Regulation of immune responses by TGF-beta. Annu Rev Immunol. 1998;16:137-61.

78. Fiorentino DF, Zlotnik A, Vieira P, Mosmann TR, Howard M, Moore KW, et al. IL-10 acts on the antigen-presenting cell to inhibit cytokine production by Th1 cells. J Immunol. 1991;146:3444-51.

79. Buelens C, Willems F, Delvaux A, Pierard G, Delville JP, Velu T, et al. Interleukin-10 differentially regulates B7-1 (CD80) and B7-2 (CD86) expression on human peripheral blood dendritic cells. Eur J Immunol. 1995;25:2668-72.
80. De Waal MR, Haanen J, Spits H, Roncarolo MG, Te VA, Figdor C, et al. Interleukin 10 (IL-10) and viral IL-10 strongly reduce antigen-specific human $T$ cell proliferation by diminishing the antigen-presenting capacity of monocytes via downregulation of class II major histocompatibility complex expression. J Exp Med. 1991;174:915-24.

81. Groux H, Bigler M, de Vries JE, Roncarolo MG. Interleukin-10 induces a long-term antigen-specific anergic state in human CD4+ T cells. J Exp Med. 1996;184:19-29.

82. Jonuleit $H$, Schmitt $E$. The regulatory $T$ cell family: distinct subsets and their interrelations. J Immunol. 2003;171:6323-7.

83. Yudoh K, Matsuno $H$, Nakazawa F, Yonezawa T, Kimura T. Reduced expression of the regulatory CD4+ T cell subset is related to Th1/Th2 balance and disease severity in rheumatoid arthritis. Arthritis Rheum. 2000;43:617-27.

84. Gutterman JU. Cytokine therapeutics: lessons from interferon alpha. Proc Natl Acad Sci U S A. 1994:91:1198-205.

85. Tompkins WA. Immunomodulation and therapeutic effects of the oral use of interferon-alpha: mechanism of action. J Interferon Cytokine Res. 1999; 19:817-28.

86. Brinkmann V, Geiger T, Alkan S, Heusser $\mathrm{CH}$. Interferon alpha increases the frequency of interferon gamma-producing human CD4+ T cells. J Exp Med. 1993;178:1655-63.

87. Akbar AN, Lord JM, Salmon M. IFN-alpha and IFN-beta: a link between immune memory and chronic inflammation. Immunol Today. 2000:21:337-42.

88. Monteleone G, Pender SL, Wathen NC, MacDonald TT. Interferon-alpha drives T cell-mediated immunopathology in the intestine. Eur J Immunol. 2001;31:2247-55. 